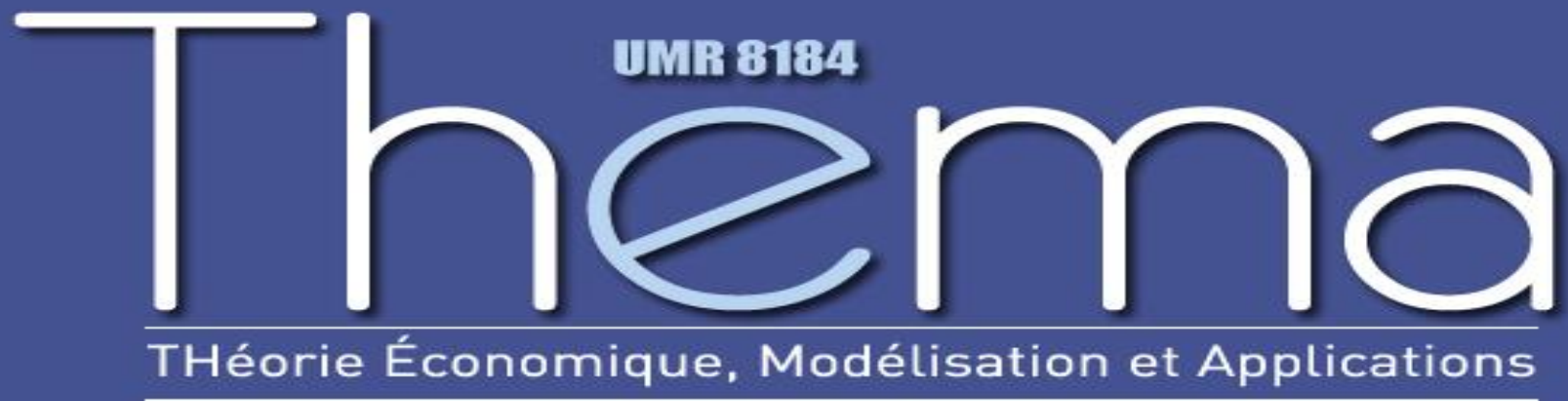

Thema Working Paper $n^{\circ}$ 2012-30

Université de Cergy Pontoise, France

Bayesian Population Dynamics of Spreading Species

Arnaud Z. Dragicevic

February, 2012 


\title{
Bayesian Population Dynamics of Spreading Species ${ }^{1}$
}

\author{
Arnaud Z. Dragicevic ${ }^{2}$
}

February 2012

\begin{abstract}
The invasion of native species by exotic species is one of the most serious threats to biodiversity and ecosystem functioning. Despite a number of empirical and theoretical studies, there is still no general model about why or when settlement becomes invasion. The purpose of this work is to test a model of Bayesian population dynamics relying on bestresponse strategies that could help in resource management and bioeconomic modeling. Given the exotic species survival probability, our static game unveils a breaking-level probability in mixed-strategies, where it is in the interest of exotic species to invade and in the interest of native species to resist. In dynamic setting, we introduce a stochastic version of the balance equation based on conditional probabilities. When the exotic species survival probability and the availability of resources in the ecosystem are respectively high and low, the dynamics shows that the convergence of subpopulations toward steady-states operates at a high pace.
\end{abstract}

Keywords: bioeconomics, best-responses, balance equation, Bayesian population dynamics, resource management, biodiversity, invasive species.

JEL Codes: C61, C62, C73, Q5, Q56, Q57

\footnotetext{
${ }^{1}$ I would like to thank Matias Nunez and Guy Meunier for our discussions and their helpful comments toward this project. The usual caveats apply.

${ }^{2}$ University of Cergy-Pontoise, THEMA, F-95000 Cergy-Pontoise, e-mail: arnaud.dragicevic@u-cergy.fr
} 


\section{Introduction}

The invasion of native species by exotic species has become one of the most serious threats to biodiversity and ecosystem functioning (Vitousek et al. 1996, Lonsdale 1999, Williamson 1999, Sebert-Cuvillier et al. 2007). Exotic species can ruin the ecological health and economic value of ecosystems (Williamson and Fitter 1996). Native species can be negatively affected by exotic species or ecosystem changes caused by exotic invaders. For example, many species listed as threatened or endangered under the Endangered Species Act are at risk because of competition with, predation by and pressures of nonnative species (Pimentel et al. 2000).

Despite a number of empirical and theoretical studies (Lonsdale 1999, Zedler and Kercher 2004, Davis et al. 2006, Meiners 2007, Sebert-Cuvillier et al. 2007), there is still no general model about why or when settlement becomes invasion. Both the attributes that make a species an invader (Kolar and Lodge 2001) and the characteristics that predispose an ecosystem to invasion (Sebert-Cuvillier et al. 2007) are still weakly understood.

What we know is that three factors promote the settlement of new species on an area: the availability of resources, the absence of natural enemies or competitors and the physical environment (Shea and Chesson 2002, Byers and Noonburg 2003). Elton (1958) spoke about the biotic resistance of ecosystems and asserted that strong interactions between native and exotic species prevent the latter from spreading. However, strong interactions can facilitate the settlement of invading species and positive interactions can increase the survival rate of exotic species and the death rate of native species (Simberloff and Von Holle 1999).

The invasive species management aims at reducing the invasion pace, in order to limit the environmental and economic impacts of invaders (Pimentel et al. 2000). The literature on this subject is substantial (Born and al. 2005, Marten and Moore 2011), but the lack of general guidelines generates a multitude of population models for each invader, as pointed out by Ramula et al. (2008). These population models work according to the demographic processes based on survival, growth and fecundity. Crawley (1986) asserted that simple demographic models are not useful given the absence of clear demographic profile of a successful invader. Nevertheless, models based on logistic population growth, where the rate of reproduction is proportional to the existing population and the amount of available resources, have emerged (see Clark 1990, Jayasuriya et al. 2011). Our approach concurs with these factors.

The invasive species establish and spread stochastically (Davis et al. 2000, Shea and Chesson, 2002). Therefore, a deterministic model is reductive, since it does not capture the 
environmental stochasticity. Although uncertainty is a central characteristic of the invasion process (Williamson 1999), the survey on biological invasion done by Born and al. (2005) indicates that uncertainty arising in the ecological context of the invasive process is not handled. Likewise, Marten and Moore (2011) emphasize that the absence of biological uncertainty in deterministic bioeconomic models leads to significant bias in management solutions. In this way, Olson and Roy (2002) show that stochastic shocks to the population growth affect the choice of management strategy.

This paper answers the calls by Born et al. (2005) and Saphores and Shogren (2005) to study exotic pests respectively in uncertainty and Bayesian framework. Along the lines of Ramula et al. (2008) who explore general patterns based on survival, and following the recent work by Bischi et al. (2009), we aim at modeling population dynamics based on bestresponses and conditional probabilities that could guide resource managers and bioeconomists. Our static game unveils pure and mixed Nash-equilibrium strategies. While not invading and not resisting are always the pure-strategy equilibria, there is a breaking-level probability in mixed strategies, where, given the exotic species survival rate, the bestresponse for exotic species is to invade and the best-response for native species is to resist. In dynamic setting, we introduce a stochastic version of the balance equation. When the exotic species survival probability is high and the availability of resources in the ecosystem is low, the Bayesian population dynamics shows that the convergence of subpopulations toward steady-states operates in rapid dynamics.

After this opening section, we begin with the static game and present the pure and mixed-strategy Nash equilibria in Section 2. Section 3 introduces the Bayesian population dynamics and discusses the stability of equilibria. Conclusive remarks are given in Section 4.

\section{Static model}

Following a work by Liu et al. (2006), let $i$ and $j$ respectively be an exotic and a native species that simultaneously interact. Let $w>0$ represent the ecosystem fundamental value, notably that of the biotope and biotic community resources, in which the two species evolve. Let $\alpha$, with $\alpha \in[0,1]$, be the rate of availability of resources in the ecosystem. The availability factor reflects the hypotheses that the spread of nonnative species depends on the resource availability in the ecosystem (Lonsdale 1999). 
Exotic species $i$ 's behavior is defined by a set of two actions: it either settles or spreads in native species $j$ 's environment. When $i$ spreads, it does it at an effort or at a cost of $c_{s} \geq 0$. Native species $j$ holds a set of two actions as well. It can either endorse the invasion or resist it at an effort or a cost of $c_{r} \geq 0$. In what follows, the cost for both species is assumed equivalent, i.e. $c_{s}=c_{r}=c$. Despite appearing strong, this assumption is rather reasonable and reflects the fact that the greater the resistance from native species, the greater the effort needful to exotic species to spread. Finally, let $\mu$, with $\mu \in[0,1]$, be the survival probability of exotic species endowed with a certain level of resilience in the habitat. The survival probability is a common prior to both players. We assume that $\alpha$ and $\mu$ are exogenous, that is, the nature decides ex ante on the rate of availability of resources and the likelihood of survival of the incomer.

\subsection{Exotic species $i$ 's payoff function}

If exotic species $i$ settles, its payoff amounts to:

- $\quad \alpha w$ when native species $j$ resists;

- $\quad w$ when native species $j$ endorses.

When native species $j$ resists, the expected payoff of exotic species $i$ is the actual value $\alpha w$ of resources. When native species $j$ endorses exotic species $i$ 's settlement without resistance, the payoff of the latter is the ecosystem fundamental value, since all resources are within reach at no cost.

If exotic species $i$ spreads, its payoff amounts to:

- $\quad(1-\alpha) w-c$ when native species $j$ resists;

- $\quad w-c$ when native species $j$ endorses.

When native species $j$ resists, the payoff of exotic species $i$ is the ecosystem residual value $(1-\alpha) w$ at the invasion $\operatorname{cost} c$. The residual value is the difference between the fundamental $w$ and actual $\alpha w$ values: exotic species $i$ values unavailable resources at a spreading cost. When native species $j$ shows no resistance to invasion, the payoff of exotic species $i$ is the ecosystem fundamental $w$ value minus the spreading cost $c$.

\subsection{Native species j's payoff function}


If native species $j$ resists, its payoff amounts to:

- $\quad(1-\alpha) w-c$ when exotic species $i$ settles;

- $\quad \alpha w-c$ when exotic species $i$ spreads.

When exotic species $i$ settles, native species $j$ 's payoff is the ecosystem residual value $(1-\alpha) w$ less the resistance cost $c$. If native species $j$ unconditionally resists, it does not share the ecosystem resources and values even what it does not consume. When exotic species $i$ spreads, native species $j$ 's payoff is the ecosystem actual value $\alpha w$ minus the resistance cost c. Given the establishment of competition over resources, native species cannot value the whole ecosystem anymore and now values what is available for its subsistence less the cost of protection.

If native species $j$ endorses, its payoff amounts to:

- $\quad w$ when exotic species $i$ settles;

- 0 when exotic species $i$ spreads.

When native species $j$ endorses, its payoff amounts to the ecosystem fundamental value $w$. As exotic species $i$ spreads and grabs resources without resistance, native species $j$ 's payoff turns out to be null, which implies that $\alpha=1$, i.e. it makes resources fully available for the spreading species.

In sum, the game matrix is as follows

\begin{tabular}{llcc}
\hline & \multicolumn{2}{c}{ Native species $j$} \\
\hline \multirow{2}{*}{ Exotic species $i$} & resists & endorses \\
\cline { 2 - 4 } & spreads & $\alpha w ;(1-\alpha) w-c$ & $w ; w$ \\
\hline
\end{tabular}

\subsection{Pure strategies}

We now analyze the Nash equilibrium based on the assumption that the exotic species survival probability $\mu$ is a common prior. When native species $j$ resists, its expected payoff amounts to 


$$
\mathrm{E}\left(\pi_{j}^{r}\right)=(1-\mu)[(1-\alpha) w-c]+\mu(\alpha w-c)
$$

When native species $j$ endorses, its expected payoff amounts to

$$
\mathrm{E}\left(\pi_{j}^{\bar{r}}\right)=(1-\mu) w+\mu 0
$$

In pure strategy, native species $j$ will resist to exotic species $i$ only if $\pi_{j}^{r}>\pi_{j}^{\bar{r}}$. Algebraically, it means that

$$
\mu>\frac{\alpha w+c}{2 \alpha w}
$$

When the probability that exotic species $i$ survives is high enough, native species $j$ will resist, regardless of whether exotic species $i$ spreads or not. Yet, if native species $j$ resists, exotic species $i$ should not invade because of the cost. Exotic species $i$ 's best response is then not to

spread. This is not a Nash equilibrium. When $\pi_{j}^{r}<\pi_{j}^{\bar{r}}$, we have $\mu<\frac{\alpha w+c}{2 \alpha w}$. Thereby, when the survival probability of exotic species $i$ is low enough, native species $j$ 's best response is not to resist, which in pure strategy is the Nash equilibrium.

Proposition 1. For any survival probability such that $\mu<\frac{\alpha w+c}{2 \alpha w}$, the Nash equilibrium corresponds to the pair of pure strategies $\{$ species $i$ settles; species $j$ endorses\}.

\subsection{Mixed strategies}

\section{4. a. Equilibrium strategy of species $i$ given the expected payoff of species $j$}

Let $p$ be the probability that exotic species $i$ spreads, and $1-p$ that it settles. Given the survival probability of species $i$, the expected payoff of native species $j$ that resists resumes to

$$
\mathrm{E}\left(\pi_{j}^{r} \mid \mu\right)=p \mu(\alpha w-c)+(1-p)[(1-\alpha) w-c]
$$

As a mixed strategy, expression (4) is the factorized sum of expected payoffs relative to: 
- The probability that exotic species $i$ spreads given its probability of survival, when native species $j$ resists.

- The probability that exotic species $i$ settles given its probability of survival, when native species $j$ resists.

- The probability that exotic species $i$ settles given its probability of death, when native species $j$ resists.

Given the survival probability of exotic species $i$, the expected payoff of native species $j$ that endorses resumes to

$$
\mathrm{E}\left(\pi_{j}^{\bar{r}} \mid \mu\right)=(1-p) w
$$

As a mixed strategy, expression (5) is the factorized sum of expected payoffs relative to:

- The probability that exotic species $i$ spreads given its probability of survival, when native species $j$ endorses.

- The probability that exotic species $i$ settles given its probability of survival, when native species $j$ endorses.

- The probability that exotic species $i$ settles given its probability of death, when native species $j$ endorses.

Equalizing $\mathrm{E}\left(\pi_{j}^{r} \mid \mu\right)=\mathrm{E}\left(\pi_{j}^{\bar{r}} \mid \mu\right)$ yields

$$
p^{*}=\frac{\alpha w+c}{\alpha w(\mu+1)-c(\mu-1)}
$$

Proposition 2. In mixed strategies, the best response for exotic species $i$ is to spread with probability $p^{*}$.

2.4.b. Equilibrium strategy of species $j$ given the expected payoff of species $i$

Let $q$ be the probability that native species $j$ resists, and $1-q$ that it endorses. The expected payoff of exotic species $i$ that settles resumes to 


$$
\mathrm{E}\left(\pi_{i}^{\bar{s}}\right)=w[q(\alpha-1)+1]
$$

As a mixed strategy, it is the sum of expected payoffs and depends on the probability that agent $j$ resists and endorses.

The expected payoff of exotic species $i$ that spreads resumes to

$$
\mathrm{E}\left(\pi_{i}^{s}\right)=w(1-q \alpha)-c
$$

Setting up $\mathrm{E}\left(\pi_{i}^{\bar{s}}\right)=\mathrm{E}\left(\pi_{i}^{s}\right)$ yields

$$
q^{*}=\frac{c}{w(1-2 \alpha)}
$$

Proposition 3. In mixed strategies, the best response for native species $j$ is to resist with probability $q^{*}$.

We can now make a broader proposition.

Proposition 4. Given the survival probability of exotic species $i$, the mixed-strategy Nash equilibrium corresponds to $\left\{\right.$ species $i$ spreads with $p^{*}$, species $j$ resists with $\left.q^{*} \mid \mu\right\}$.

\section{Dynamic model}

Understanding population attributes of invasive species is a prerequisite to manage invasions efficiently (Sebert-Cuvillier et al. 2007). For that reason, let us carry out an evolutionary analysis over the species' spreading. Species can invade and resist at any time. Since species do not have knowledge of the underlying structure of the game, we assume that the switching mechanism takes place according to a social learning mechanism (Ellison and Fudenberg 1995, Hofbauer and Sigmund 1998, Bischi et al. 2009). It is now admitted that social learning generates imitation and protoculture. As well, we know that ecological selection favors successful strategies which percolate in time (Hofbauer and Sigmund 1998).

Following a work by Bischi et al. (2009), we assume that a species samples a species that has chosen the opposite strategy in the past. At each time period, if the payoff of the 
sampled species is greater than its own, the latter switches to this strategy. The sampling follows the uniform probability law so the probability of comparing payoffs with species of a given strategy is proportional to the fraction of species using that strategy.

Let the population of exotic species be divided in fractions of spreaders $s(t)$ and settlers $\bar{s}(t)$ operating in period $t$, where $s(t)+\bar{s}(t)=1$. In parallel, let the population of native species be divided in fractions of resistants $r(t)$ and endorsers $\bar{r}(t)$ operating at $t$, with $r(t)+\bar{r}(t)=1$. The sum denotes a normalized subpopulation density such that 0 corresponds to extinction and 1 is the maximal subpopulation density.

\subsection{Spreading dynamics of exotic species}

The probability to switch from spreading to settlement is $\rho_{s \bar{s}}$. This probability is obtained by multiplying the probability that the spreading fraction $s$ measures up its payoff against a settling fraction $\bar{s}$ with the probability that the payoff of that fraction is greater than its own, i.e. $\operatorname{Pr}\left(\pi_{i}^{\bar{s}}>\pi_{i}^{s}\right)$. We thus have

$$
\rho_{s \bar{s}}=\bar{s} \operatorname{Pr}\left(\pi_{i}^{\bar{s}}>\pi_{i}^{s}\right)=(1-s) \operatorname{Pr}\left(\pi_{i}^{\bar{s}}>\pi_{i}^{s}\right)
$$

Given that the native species equilibrium strategy equates exotic species expected payoffs at $q^{*}$, it implies that $\operatorname{Pr}\left(\pi_{i}^{\bar{s}}>\pi_{i}^{s}\right)=\tilde{q}$, with $\tilde{q} \geq q^{*}$. Therefore, we have $\rho_{s \bar{s}}=(1-s) \tilde{q}$.

Symetrically, we have $\rho_{\bar{s} s}$ such that

$$
\rho_{\overline{s s}}=s \operatorname{Pr}\left(\pi_{i}^{s}>\pi_{i}^{\bar{s}}\right)
$$

Given that the native species equilibrium strategy equates the exotic species expected payoffs at $q^{*}$, it implies that $\operatorname{Pr}\left(\pi_{i}^{s}>\pi_{i}^{\bar{s}}\right)=1-\tilde{q}$, with $1-\tilde{q}<q^{*}$. We have $\rho_{\bar{s} s}=s(1-\tilde{q})$.

The dynamic equation describing the expected fraction of spreaders among exotic species is given by

$$
s(t+1)=s(t)+[1-s(t)] \operatorname{Pr}\left[\rho_{\overline{s s}} \mid s(t)\right]-s(t) \operatorname{Pr}\left[\rho_{s \bar{s}} \mid 1-s(t)\right]
$$


The fraction of spreading species at time $t+1$ is the sum of the fraction of spreaders at time $t$, i.e. $s(t)$, and of the product of the fraction of settlers and the conditional probability of switching strategy given the fraction of spreaders at time $t$, i.e. $[1-s(t)] \operatorname{Pr}\left[\rho_{\overline{s s}} \mid s(t)\right]$, minus the fraction of spreaders multiplied by the conditional probability of switching strategy given the fraction of settlers at time $t$, i.e. $s(t) \operatorname{Pr}\left[\rho_{s \bar{s}} \mid 1-s(t)\right]$. Equation (12) can be interpreted as a variant of the balance equation where species change strategies in light of conditional probabilities but cannot appear nor disappear from nowhere.

We follow the rationale of population growth models, but the novelty of our dynamic balancing stands for the probability of switching strategy conditional on the fraction of species which strategy is being benchmarked. We thus term it Bayesian population dynamics $^{3}$. Indeed, it is apt that an entity will be prone to the mass effect, that is, it will switch strategy in proportion to the fraction of entities in the population already using that strategy. Rewritten, the precedent equation yields

$$
s(t+1)=[2 s(t)]\left(\tilde{q}^{2}-\tilde{q}\right)-\tilde{q}^{2}+2 \tilde{q}
$$

\subsection{Resistance dynamics of native species}

The probability of switching from resistance to endorsement is $\rho_{r \bar{r}}$. This probability is obtained by the product of the probability that the resistant fraction $r$ measures up its payoff against an endorsing fraction $\bar{r}$ and the probability that the payoff of that fraction is greater than its own, i.e. $\operatorname{Pr}\left(\pi_{i}^{\bar{r}}>\pi_{i}^{r}\right)$. We thus have

$$
\rho_{r \bar{r}}=\bar{r} \operatorname{Pr}\left(\pi_{j}^{\bar{r}}>\pi_{j}^{r}\right)=(1-r) \operatorname{Pr}\left(\pi_{j}^{\bar{r}}>\pi_{j}^{r}\right)
$$

Given that the exotic species equilibrium strategy equates the native species expected payoffs at $p^{*}$, it implies that $\operatorname{Pr}\left(\pi_{j}^{\bar{r}}>\pi_{j}^{r}\right)=\tilde{p}$, with $\tilde{p} \leq p^{*}$. Therefore, we have $\rho_{r \bar{r}}=(1-r) \tilde{p}$.

Symmetrically, we have $\rho_{\bar{r} r}$ such that

$$
\rho_{\bar{r} r}=r \operatorname{Pr}\left(\pi_{j}^{r}>\pi_{j}^{\bar{r}}\right)
$$

\footnotetext{
${ }^{3}$ Alternative Bayesian density-dependant growth model has been studied by Frigessi et al. (2005)
} 
Given that the exotic species equilibrium strategy equates the native species expected payoffs at $p^{*}$, it implies that $\operatorname{Pr}\left(\pi_{j}^{r}>\pi_{j}^{\bar{r}}\right)=1-\tilde{p}$, with $1-\tilde{p}>p^{*}$. Therefore, we have $\rho_{\bar{r} r}=r(1-\tilde{p})$.

The dynamic equation describing the expected fraction of resistants among native species is given by

$$
r(t+1)=r(t)+[1-r(t)] \operatorname{Pr}\left[\rho_{\bar{r} r} \mid r(t)\right]-r(t) \operatorname{Pr}\left[\rho_{r \bar{r}} \mid 1-r(t)\right]
$$

The fraction of resisting species at time $t+1$ is the sum of the fraction of resistants at time $t$, i.e. $r(t)$, and of the product of the fraction of endorsers and the conditional probability of switching strategy given the fraction of resistants at time $t$, i.e. $[1-r(t)] \operatorname{Pr}\left[\rho_{\overline{r r}} \mid r(t)\right]$, minus the fraction of resistants multiplied by the conditional probability of switching strategy given the fraction of endorsers at time $t$, i.e. $r(t) \operatorname{Pr}\left[\rho_{r \bar{r}} \mid 1-r(t)\right]$. Rewritten, the Bayesian population dynamics yields

$$
r(t+1)=[2 r(t)]\left(\tilde{p}^{2}-\tilde{p}\right)-\tilde{p}^{2}+2 \tilde{p}
$$

\subsection{Dynamical system}

Fractions of spreading and resistant species in time are subject to a nonlinear system. The dynamical system $D(t+1)$ can be described by the following map in $\mathbb{R}^{2}$ in the dynamic variables $s$ and $r$ :

$$
D(t+1):\left\{\begin{array}{l}
s(t+1)=[2 s(t)]\left(\tilde{q}^{2}-\tilde{q}\right)-\tilde{q}^{2}+2 \tilde{q} \\
r(t+1)=[2 r(t)]\left(\tilde{p}^{2}-\tilde{p}\right)-\tilde{p}^{2}+2 \tilde{p}
\end{array}\right.
$$

A steady state of the dynamical system is a point $S=\left(s^{*}, r^{*}\right)$ satisfying the following nonlinear system of equations

$$
\left\{\begin{array}{l}
(2 s-1)\left(\tilde{q}^{2}-\tilde{q}\right)=0 \\
(2 r-1)\left(\tilde{p}^{2}-\tilde{p}\right)=0
\end{array}\right.
$$


Any population configuration is represented by the pair of subpopulation densities of exotic and native species. The system has four corner equilibria $S=(0,0), S=(0,1)$, $S=(1,0), S=(1,1)$ and an inner equilibrium $S=\left(s^{*}, r^{*}\right)$. All corner equilibria represent null or full equilibria and can be easily interpreted. For example, $S=(1,0)$ means that the all entities among exotic species spread and no entity among native species resists. The inner equilibrium signifies that certain levels of density of the subpopulations spread and resist.

Let us now study the necessary and sufficient conditions for a unique inner equilibrium to exist. The conditions are derived in Tables 1 and 2 . As we set $s(t+1):=f(s)$ and $r(t+1):=g(r)$, we obtain

Table 1 Inner equilibrium conditions for $f(s)$

\begin{tabular}{ccc}
\hline$f(0)<0$ & $f(1)>0$ & $f^{\prime}(s)>0$ \\
\hline$\alpha \in\left[0, \frac{1}{2}\right)$ & $\alpha \in\left[0, \frac{1}{2}\right)$ & $\alpha \in\left[0, \frac{1}{2}\right)$ \\
$c>0, w \in\left(0,-\frac{c}{4 \alpha-2}\right)$ & $c>0$ & $c>0, w \in\left(0,-\frac{c}{2 \alpha-1}\right)$ \\
\hline$\alpha \in\left(\frac{1}{2}, 1\right]$ & $\alpha \in\left(\frac{1}{2}, 1\right]$ & $\alpha \in\left(\frac{1}{2}, 1\right]$ \\
$c>0$ & $c>0$ & $c>0$ \\
\hline
\end{tabular}

Table 2 Inner equilibrium conditions for $g(r)$

\begin{tabular}{ccc}
\hline$g(0)<0$ & $g(1)>0$ & $g^{\prime}(r)>0$ \\
\hline$\alpha>0, c>0$ & $\alpha=0, c>0$ & $\alpha=0, c>0$ \\
$\mu \in\left(\frac{1}{2}, 1\right]$ & $\mu \in(0,1]$ & $\mu \in(0,1)$ \\
\hline$\alpha \in(0,1], c>0$, & $\alpha \in[0,1)$ & $\alpha \in(0,1], c>0$ \\
$w \in\left(0, \frac{c}{3 \alpha}\right), \mu \in\left(\frac{\alpha w+c}{2 c-2 \alpha w}, 1\right]$ & $\mu \in[0,1]$ & $w \in\left(0, \frac{c}{\alpha}\right), \mu \in(0,1]$ \\
\hline
\end{tabular}

The roots of $f(s)=0$ and $g(r)=0$ are equal to

$$
\left\{\begin{array}{l}
s^{*}=\frac{\tilde{q}-2}{2(\tilde{q}-1)} \\
r^{*}=\frac{\tilde{p}-2}{2(\tilde{p}-1)}
\end{array}\right.
$$


Given that $s^{*}, r^{*} \in(0,1)$, the inner equilibrium exists for

\begin{tabular}{cccc}
\hline \multicolumn{2}{c}{$s^{*} \in(0,1)$} & $r^{*} \in(0,1)$ \\
\hline$\alpha \in\left[0, \frac{1}{2}\right)$ & $\alpha \in\left(\frac{1}{2}, 1\right]$ & $\alpha=0, c>0$ & $\alpha \in(0,1], w \in\left(0, \frac{c}{\alpha}\right)$ \\
$w \in\left(0,-\frac{c}{2 \alpha-1}\right)$ & $\mu \in\left(\frac{1}{2}, 1\right]$ & $\mu \in(0,1]$ \\
\hline
\end{tabular}

Proposition 5. There exists a unique inner equilibrium $S=\left(s^{*}, r^{*}\right)$.

The stability of equilibria is then studied by means of the linearization analysis (see Appendix). Eigenvalues $\lambda$ of the Jacobian matrix are outlined in Table 3.

Table 3 Eigenvalues of the Jacobian matrix

\begin{tabular}{lccccc}
\hline$S=(s, r)$ & $(0,0)$ & $(0,1)$ & $(1,0)$ & $(1,1)$ & $\left(s^{*}, r^{*}\right)$ \\
\hline stable & $\alpha \in\left(0, \frac{1}{2}\right)$ & & $\alpha \in[0,1]$ & $\alpha \in(0,1]$ & $\alpha \in\left[\frac{2}{5}, 1\right]$ \\
$(\lambda<0)$ & $\mu \in(0,1]$ & & $\mu \in(0,1]$ & $\mu \in(0,1]$ & $\mu \in(0,1]$ \\
\hline unstable & $\alpha \in\left(\frac{1}{2}, 1\right]$ & $\alpha \in(0,1]$ & & $\alpha=0$ & $\alpha \in\left[0, \frac{2}{5}\right)$ \\
$(\lambda>0)$ & $\mu \in(0,1]$ & $\mu \in(0,1]$ & & $\mu \in(0,1]$ & $\mu \in(0,1]$ \\
\hline
\end{tabular}

While the equilibrium in which all exotic species spread and no native species resists is always dynamically stable, the equilibrium where no exotic species spreads and all native species resist is always instable. Apart these two asymmetric cases, the symmetric corner equilibria and the inner equilibrium display both stable and unstable configurations, depending on the values of parameters.

Patten (2010) clarifies that the useful steady-state question is not whether steady-states are achieved in ecosystems, but rather, whether there is a directing tendency that organizes the succession process. Our results give intuition that a directing tendency could lie in the availability of resources in the ecosystem for which species compete and the survival probability of nonnative species.

Proposition 6. The corner equilibrium $S=(1,0)$ is always stable. The corner equilibrium $S=(0,1)$ is always unstable. The inner $S=\left(s^{*}, r^{*}\right)$ and corner $S=(0,0)$ and $S=(1,1)$ equilibria can be stable or unstable, depending on the parameters' values. 


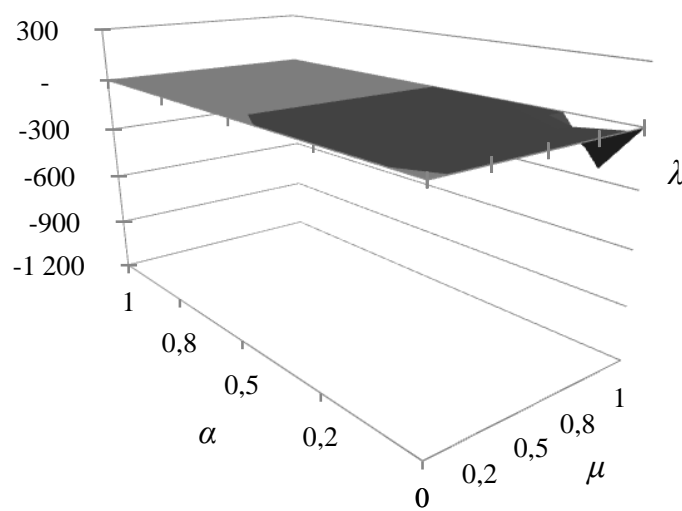

a. $S=(0,0)$

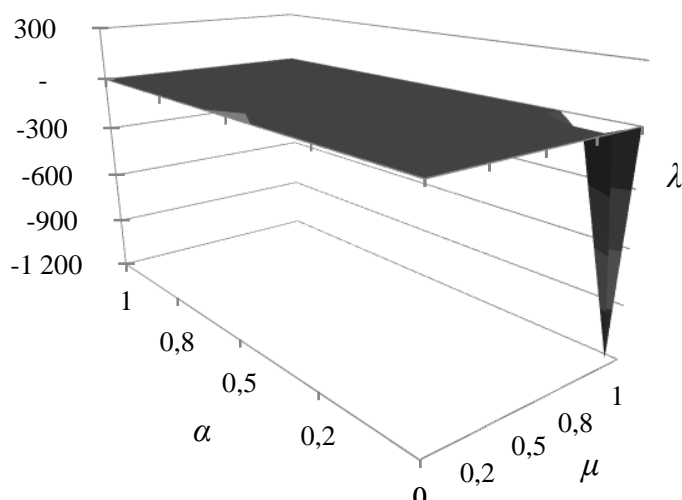

c. $S=(1,0)$

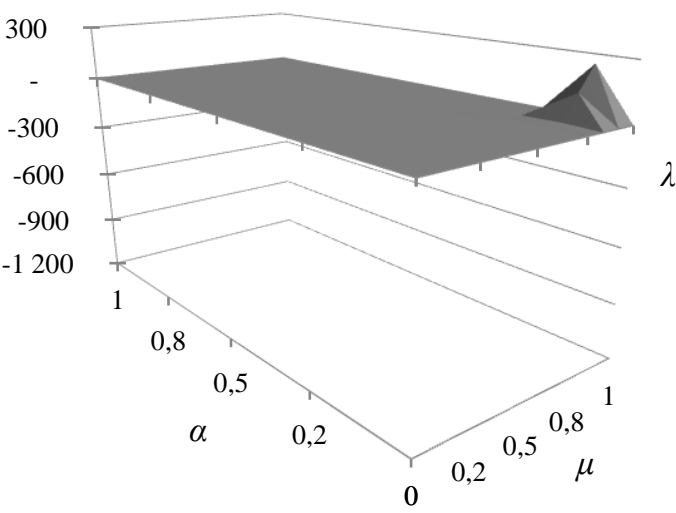

b. $S=(0,1)$

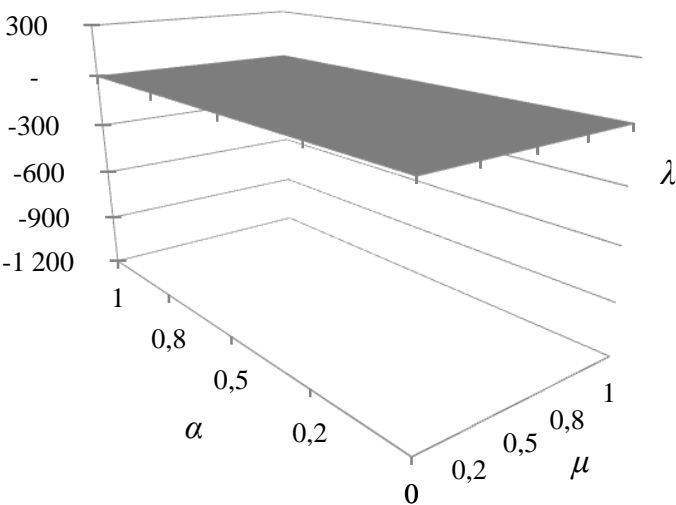

d. $\quad S=(1,1)$

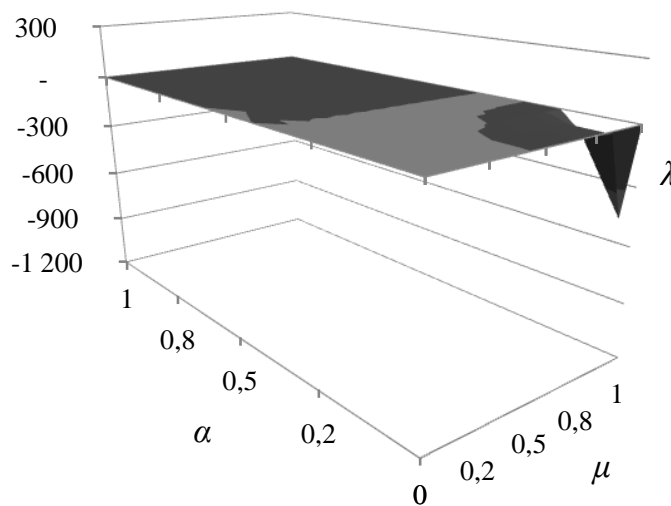

e. $S=\left(s^{*}, r^{*}\right)$ for $s^{*}=r^{*}=0.3$

Fig. 1 Numerical simulations of $\lambda$ given $\alpha$ and $\mu$, for $c=\frac{1}{2} w$ 
Figures 1.a., 1.b., 1.c., 1.d. and 1.e. illustrate the stability of equilibria with regard to the parameters' values (Tables 4-8 in Appendix). In addition to the nature of steady-states provided by $\lambda$, the eigenvalues also provide information on the pace of convergence toward steady-states. Numerical simulations that we conducted under the linearization analysis reveal that all configurations show the same distribution of convergence pace, regardless of the stationarity in $S$. Roughly $95 \%$ of cases reveal a low rate of convergence, for $\lambda= \pm 0.00$, and this is verified for nearly all levels of availability of resources in the ecosystem and all rates of survival of exotic species.

The only zone where the convergence pace suddenly becomes high and steady-states rapidly occur is when the survival rate of the exotic species is almost certain, i.e. $\lim (\mu)=1$, and resources are very scarce, i.e. $\lim (\alpha)=0$. This particularity is verified for both corner and inner equilibria for $r^{*}<\frac{1}{2}$ and can be easily justified. When exotic species are highly likely to survive in an environment where resources are in short supply, a strong competition with the resisting native biotic community over the resources engages. This in turn provokes a rapid steady-state population balancing.

Proposition 7. When the exotic species survival rate and the availability of resources in the ecosystem are respectively high and low, the population convergence toward steady-states operates at a high pace.

Proposition 7 excludes $r^{*} \geq \frac{1}{2}$. Indeed, as the condition $w \in\left(0, \frac{c}{\alpha}\right)$ is unverified, there is no inner equilibrium for this value of initial density. The condition states that the inner equilibrium exists when the fundamental value of resources is at least as great as the cost of resisting for its sake. As a result, corner equilibria with null- or full-density configurations of resistants ensue perforce.

Proposition 8. When $r^{*} \geq \frac{1}{2}$ and $w \notin\left(0, \frac{c}{\alpha}\right)$, resistants evolve toward corner equilibria.

\section{Conclusion}

As explicated in the introduction, there is still no global model about why or when an invasion occurs. We try to give an answer to this shortage by means of game theory and 
Bayesian population dynamics, using best-responses and a balance equation dependent on conditional probabilities. Our models yield some quite intuitive results, such as invasion as best-response strategy and strong competition between exotic and native species over scarce resources in rapid dynamics. It should not be intricate for bioeconomists or resource managers to model or simulate a biotope evolution and to use our results for their decision-making.

To its detriment, our framework does not take into account the traits of exotic species other than the resistance potential - life cycle or behavioral aspects of reproduction - and abiotic components of the environment - light, temperature, water, gases or soil properties -, which complexify the interactions between native and exotic species (Williamson and Fitter 1996). Nevertheless, its minimalism and Bayesian approach enable to track population dynamics by giving the subpopulation model-species the proper payoff functions.

\section{References}

Bischi, G.-I., Lamantia, F. and Sbragia, L. (2009), "Strategic Interaction and Imitation Dynamics in Patch Differentiated Exploitation of Fisheries", Ecological Complexity, 6: 353-362.

Born, W., Rauschmayer, F. and Bräuer, I. (2005), "Economic Evaluation of Biological Invasions - A Survey", Ecological Economics, 55: 321-336.

Byers, J. and Noonburg, E. (2003), "Scale Dependent Effects of Biotic Resistance to Biological Invasion", Ecology, 84: 1428-1433.

Clark, C. (1990), "Mathematical Bioeconomics: The Optimal Management of Renewable Resources", New York, USA: Wiley.

Davis, S., Landis, A., Nuzzo, V., Blossey, B., Gerber, E. and Hinz, L. (2006), "Demographic Models Inform Selection of Biocontrol Agents for Garlic Mustard (Alliaria Petiolata)", Ecological Applications, 16: 2399-2410.

Ellison, G. and Fudenberg, D. (1995), "Word-of-Mouth Communication and Social Learning", Quarterly Journal of Economics, 110: 93-125.

Elton, C. (1958), "The ecology of invasions by animals and plants", New York, USA: Wiley.

Frigessi, A., Holden, M., Marshall, C., Viljugrein, H., Stenseth, N., Holden, L., Ageyev, V. and Klassovskiy, N. (2005), "Bayesian Population Dynamics of Interacting Species: Great Gerbils and Fleas in Kazakhstan", Biometrics, 61: 230-238.

Hofbauer, J., and Sigmund, K. (1998), "Evolutionary Games and Population Dynamics", Cambridge University Press.

Kolar, C. (2001), "Progress in Invasion Biology: Predicting Invaders", Trends in Ecology and Evolution, 16: 199-204.

Jayasuriya, R., Jones, R. and Van de Ven, R. (2011), "A Bioeconomic Model for Determining the Optimal Response Strategies for a New Weed Incursion", Journal of Bioeconomics, 13: 45-72.

Liu, Y., Comaniciu, C. and Man, H. (2006), "A Bayesian game approach for intrusion detection in wireless ad hoc networks", Proceeding from the 2006 Workshop on Game theory for Communications and Networks.

Lonsdale, W. (1999), "Global Patterns of Plant Invasions and the Concept of Invasibility", Ecology, 80: 1522-1536. 
Marten, A. and Moore, C. (2011), "An Option Based Bioeconomic Model for Biological and Chemical Control of Invasive Species", Ecological Economics, 88: 1098-1104.

Meiners, S. (2007), "Native and Exotic Plant Species Exhibit Similar Population Dynamics during Succession", Ecology, 88: 1098-1104.

Olson, L. and Roy, S. (2002), "The Economics of Controlling a Stochastic Biological Invasion", American Journal of Agricultural Economics, 84: 1311-1316.

Patten, B. (2010), "Natural Ecosystem Design and Control Imperatives for Sustainable Ecosystem Services", Ecological Complexity, 7: 282-291.

Pimental, D., Lach, L., Zuniga, R. and Morrison, D. (2000), "Environmental and Economic Costs of Nonindigenous Species in the United States", Bioscience, 50: 53-65.

Ramula, S., Knight, T., Burns, J. and Buckley, Y. (2008), "General Guidelines for Invasive Plant Management based on Comparative Demography of Invasive and Native Plant Populations", Journal of Applied Ecology, 45: 1124-1133.

Sebert-Cuvillier, E., Paccaut, F., Chabrerie, O., Endels, P., Goubet, O. and Decocq, G. (2007), "Local Population Dynamics of an Invasive Tree Species with a Complex Life-History Cycle: A Stochastic Matrix Model", Ecological Modelling, 201: 127-143.

Shea, K. and Chesson, P. (2002), "Community Ecology Theory as a Framework for Biological Invasions", Trends in Ecology and Evolution, 17: 170-176.

Saphores, J-D. and Shogren, J. (2005), "Managing Exotic Pests under Uncertainty: Optimal Control Actions and Bioeconomic Investigations", Ecological Economics, 52: 327339.

Simberloff, D. and Von Holle, B. (1999), "Positive Interactions of Non Indigenous Species: Invasional Meltdown?, Biological Invasions, 1: 21-32.

Vitousek, P., D’Antonio, C., Loope, L. and Westbrooks, R. (1996), "Biological Invasions as Global Environmental Change", American Scientist, 84: 218-228.

Williamson, M. and Fitter, A. (1996), "The Varying Success of Invaders", Ecology, 77: 16611666.

Williamson, M. (1999), "Invasions", Ecography, 22: 5-12.

Zedler, J. and Kercher, S. (2004), "Causes and Consequences of Invasive Plants in Wetlands: Opportunities, opportunists and Outcomes", Critical Reviews in Plant Sciences, 23: 431-452.

\section{Appendix}

\section{Equation 13}

$$
\begin{aligned}
s(t+1) & =s(t)+[1-s(t)] \operatorname{Pr}\left[\rho_{\overline{s s}} \mid s(t)\right]-s(t) \operatorname{Pr}\left[\rho_{s \bar{s}} \mid 1-s(t)\right] \\
& =s(t)+[1-s(t)] \operatorname{Pr}[s(t)(1-\tilde{q}) \mid s(t)]-s(t) \operatorname{Pr}[[1-s(t)] \tilde{q} \mid 1-s(t)] \\
& =s(t)+[1-s(t)] \frac{\operatorname{Pr}[s(t)(1-\tilde{q}) \wedge s(t)]}{\operatorname{Pr}[s(t)]}-s(t) \frac{\operatorname{Pr}[[1-s(t)] \tilde{q} \wedge 1-s(t)]}{\operatorname{Pr}[1-s(t)]} \\
& =s(t)+[1-s(t)] \frac{\int_{1-\tilde{q}}^{1} s(t) d s(t)}{\int_{0}^{1} s(t) d s(t)}-s(t) \frac{\int_{\tilde{q}}^{1} 1-s(t) d[1-s(t)]}{\int_{0}^{1} 1-s(t) d[1-s(t)]} \\
& =s(t)+[1-s(t)] \frac{-\frac{1}{2}(\tilde{q}-2) \tilde{q}}{\frac{1}{2}}-s(t) \frac{\frac{1}{2}\left(1-\tilde{q}^{2}\right)}{\frac{1}{2}} \\
& =s(t)-[1-s(t)](\tilde{q}-2) \tilde{q}-s(t)\left(1-\tilde{q}^{2}\right) \\
& =2 s(t)\left[\tilde{q}^{2}-\tilde{q}\right]-\tilde{q}^{2}+2 \tilde{q}
\end{aligned}
$$




\section{Proof of Proposition 6}

We first look at the derivatives over the availability of resources and the cost.

$$
\begin{array}{ll}
\left.\frac{\partial}{\partial \alpha} f(s)\right|_{s \in S}=\frac{4 c s}{w(1-2 \alpha)^{2}} & \left.\frac{\partial}{\partial \alpha} g(r)\right|_{r \in S}=\frac{4 c \mu w[\alpha w(\mu-\mu r+r)+c(-\mu+\mu r+r)]}{[c(\mu-1)-\alpha(\mu+1) w]^{3}} \\
\left.\frac{\partial}{\partial c} f(s)\right|_{s \in S}=\frac{2 s}{w(1-2 \alpha)} & \left.\frac{\partial}{\partial c} g(r)\right|_{r \in S}=-\frac{4 \alpha \mu w[\alpha w(\mu-\mu r+r)+c(-\mu+\mu r+r)]}{[c(\mu-1)-\alpha(\mu+1) w]^{3}}
\end{array}
$$

Eigenvalues of the Jacobian matrix for the dynamical system $D(t+1)$ of five steady-state configurations $S$ yield as follows

- For $S=(0,0)$

$$
J(\alpha, c)=\left[\begin{array}{cc}
0-\lambda & 0 \\
\frac{4 c \mu w(\alpha w \mu-c \mu)}{[c(\mu-1)-\alpha(\mu+1) w]^{3}} & -\frac{4 \alpha \mu w(\alpha w \mu-c \mu)}{[c(\mu-1)-\alpha(\mu+1) w]^{3}}-\lambda
\end{array}\right]
$$

So

$$
\operatorname{det}[J(\alpha, c)]=(0-\lambda)\left[-\frac{4 \alpha \mu w(\alpha w \mu-c \mu)}{[c(\mu-1)-\alpha(\mu+1) w]^{3}}-\lambda\right]
$$

and

$$
(0-\lambda)\left[-\frac{4 \alpha \mu w(\alpha w \mu-c \mu)}{[c(\mu-1)-\alpha(\mu+1) w]^{3}}-\lambda\right]=0
$$

Which gives

$$
\lambda=0 \text { or } \lambda=-\frac{4 \alpha \mu w(\alpha w \mu-c \mu)}{[c(\mu-1)-\alpha(\mu+1) w]^{3}}
$$

Given $(0,0), \lambda<0$ resumes to verifying

$$
-4 \alpha \mu w(\alpha w \mu-c \mu)>0 \square
$$


Table 4 Eigenvalues of the Jacobian matrix with $c=\frac{1}{2} w$ for $S=(0,0)$

\begin{tabular}{|c|c|c|c|c|c|c|c|c|c|c|c|c|c|}
\hline$/ \mu$ & 00 & 01 & .10 & .20 & .30 & .40 & .50 & .60 & .70 & 80 & 0.90 & 0.99 & 1.00 \\
\hline 000 & 00 & 00 & 0 & 000 & 0.00 & 00 & 00 & 000 & 0 & 0 & 00 & 00 & 0 \\
\hline 01 & 00 & .00 & .00 & .00 & .00 & -0.01 & 03 & 07 & 21 & 76 & 33 & 24 & 45 \\
\hline 10 & 0.00 & 0.00 & -0.00 & 0.00 & 0.01 & -0.03 & -0.06 & -0.12 & -0.24 & -0.47 & -0.94 & -1.85 & -2.00 \\
\hline 20 & 0.00 & -0.00 & 0 & 0 & -0.01 & 2 & 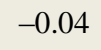 & -0.06 & 10 & -0.16 & -0.24 & -0.36 & -0.38 \\
\hline 30 & 0.00 & -0.00 & -0.00 & -0.00 & -0.01 & -0.01 & -0.02 & -0.03 & -0.04 & -0.06 & -0.08 & -0.11 & -0.1 \\
\hline 40 & 0.00 & -0.00 & -0.00 & -0.00 & -0.00 & -0.00 & -0.01 & -0.01 & -0.01 & -0.02 & -0.02 & -0.03 & -0.03 \\
\hline 0.50 & 0.00 & 000 & 0.00 & 00 & 08 & 08 & 0,00 & 0.00 & 000 & 0 & 0. & 0. & 0.0 \\
\hline 0.60 & 0.0 & .00 & +0.00 & 0.00 & 0 & as & + & + & م & +0.01 & +0 & + & 0 \\
\hline 0.70 & 0.00 & +0.00 & +0.00 & +0.00 & +0.00 & +0.00 & +0.01 & +0.01 & +0.01 & +0.01 & +0.02 & +0.02 & +0.02 \\
\hline 80 & 0.00 & +0.00 & +0.00 & +0.00 & +0.00 & +0.01 & +0.01 & +0.01 & +0.01 & +0.02 & +0.02 & +0.02 & +0.02 \\
\hline 0.90 & 0.00 & +0.00 & +0.00 & 0.00 & 0.00 & +0.01 & +0 . & +0. & م م+ & +0.02 & +0.02 & +0.02 & +0 \\
\hline 99 & 0.00 & +0.00 & +0.00 & +0.00 & +0.00 & +0.01 & +0.01 & +0.01 & +0.02 & +0.02 & +0.02 & +0.02 & +0.02 \\
\hline .0 & 0.00 & +0.00 & +0.00 & 0.00 & +0.00 & +0.01 & +0.01 & +0.01 & +0.02 & +0.02 & +0.02 & +0.02 & +0.03 \\
\hline
\end{tabular}


- For $S=(0,1)$

$$
J(\alpha, c)=\left[\begin{array}{cc}
0-\lambda & 0 \\
\frac{4 c \mu w(\alpha w+c)}{[c(\mu-1)-\alpha(\mu+1) w]^{3}} & -\frac{4 \alpha \mu w(\alpha w+c)}{[c(\mu-1)-\alpha(\mu+1) w]^{3}}-\lambda
\end{array}\right]
$$

So

$$
\operatorname{det}[J(\alpha, c)]=(0-\lambda)\left[-\frac{4 \alpha \mu w(\alpha w+c)}{[c(\mu-1)-\alpha(\mu+1) w]^{3}}-\lambda\right]
$$

And

$$
(0-\lambda)\left[-\frac{4 \alpha \mu w(\alpha w+c)}{[c(\mu-1)-\alpha(\mu+1) w]^{3}}-\lambda\right]=0
$$

Which gives

$$
\lambda=0 \text { or } \lambda=-\frac{4 \alpha \mu w(\alpha w+c)}{[c(\mu-1)-\alpha(\mu+1) w]^{3}}
$$

Given $(0,1), \lambda<0$ resumes to verifying

$$
-4 \alpha \mu w(\alpha w+c)<0 \square
$$


Table 5 Eigenvalues of the Jacobian matrix with $c=\frac{1}{2} w$ for $S=(0,1)$

\begin{tabular}{|c|c|c|c|c|c|c|c|c|c|c|c|c|c|}
\hline$/ \mu$ & 0.00 & 0.01 & 0.10 & 0.20 & 0.30 & 0.40 & 0.50 & 0.60 & 0.70 & 0.80 & 0.90 & 0.99 & 1. \\
\hline 0.00 & 0.00 & 0.00 & 0.0 & 0.00 & 0.00 & 0.00 & 0.00 & 0.00 & 0.00 & 0.00 & 0.00 & 0.00 & \\
\hline 0.01 & 0.00 & +0.00 & +0.00 & +0.01 & 0.01 & +0.03 & +0.05 & +0.12 & 0.31 & +0.99 & +5.59 & +130 & +255 \\
\hline 0.10 & 0.00 & +0.00 & +0.01 & +0.03 & +0.07 & +0.11 & +0.19 & +0.31 & +0.51 & +0.87 & +1.56 & +2.80 & +3.00 \\
\hline .20 & 0.00 & +0.00 & +0.02 & +0.04 & +0.07 & +0.11 & +0.17 & +0.24 & +0.33 & +0.46 & +0.63 & +0.85 & +0.88 \\
\hline 0.30 & 0.00 & +0.00 & +0.02 & +0.04 & +0.07 & +0.10 & +0.14 & +0.18 & +0.23 & +0.29 & +0.36 & +0.44 & +0.44 \\
\hline 40 & 0.00 & +0.00 & +0.02 & +0.04 & +0.07 & +0.09 & +0.12 & +0.15 & +0.18 & +0.21 & +0.24 & +0.28 & +0.28 \\
\hline .50 & 0.00 & +0.00 & +0.02 & +0.04 & +0.06 & +0.08 & +0.10 & +0.12 & +0.14 & +0.16 & +0.18 & +0.20 & +0.20 \\
\hline 60 & 0.00 & +0.00 & .02 & 4 & 5 & 0 & +0 & +0 & 2 & +0.13 & +0.14 & +0 & 0.15 \\
\hline 0.10 & 0.00 & +0.00 & +0.02 & +0.04 & +0.05 & +0.06 & +0.08 & +0.09 & +0.10 & +0.11 & +0.12 & +0.12 & +0.12 \\
\hline .80 & 0.00 & +0.00 & +0.02 & +0.03 & +0.05 & +0.06 & +0.07 & +0.08 & +0.08 & +0.09 & +0.10 & +0.10 & +0.10 \\
\hline 00 & 0.00 & +0.00 & +0.02 & +0.03 & +0.04 & +0.05 & +0.06 & +0.07 & +0.07 & +0.08 & +0.08 & +0.09 & +0.09 \\
\hline 93 & 0.00 & +0.00 & +0.02 & +0.03 & +0.04 & +0.05 & +0.06 & +0.06 & +0.07 & +0.07 & +0.07 & +0.08 & +0.08 \\
\hline 1.00 & 0.00 & +0.00 & +0.02 & +0.03 & +0.04 & +0.05 & +0.06 & +0.06 & +0.07 & +0.07 & +0.07 & +0.07 & +0.08 \\
\hline
\end{tabular}


- For $S=(1,0)$

$$
J(\alpha, c)=\left[\begin{array}{cc}
\frac{4 c}{w(1-2 \alpha)^{2}}-\lambda & \frac{2}{w(1-2 \alpha)} \\
\frac{4 c \mu w(\alpha w \mu-c \mu)}{[c(\mu-1)-\alpha(\mu+1) w]^{3}} & -\frac{4 \alpha \mu w(\alpha w \mu-c \mu)}{[c(\mu-1)-\alpha(\mu+1) w]^{3}}-\lambda
\end{array}\right]
$$

So

$\operatorname{det}[J(\alpha, c)]=\left[\frac{4 c}{w(1-2 \alpha)^{2}}-\lambda\right]\left[-\frac{4 \alpha \mu w(\alpha w \mu-c \mu)}{[c(\mu-1)-\alpha(\mu+1) w]^{3}}-\lambda\right]-\left[\frac{2}{w(1-2 \alpha)}\right]\left[\frac{4 c \mu w(\alpha w \mu-c \mu)}{[c(\mu-1)-\alpha(\mu+1) w]^{3}}\right]$

And

$$
\left[\frac{4 c}{w(1-2 \alpha)^{2}}-\lambda\right]\left[-\frac{4 \alpha \mu w(\alpha w \mu-c \mu)}{[c(\mu-1)-\alpha(\mu+1) w]^{3}}-\lambda\right]-\left[\frac{2}{w(1-2 \alpha)}\right]\left[\frac{4 c \mu w(\alpha w \mu-c \mu)}{[c(\mu-1)-\alpha(\mu+1) w]^{3}}\right]=0
$$

Which gives

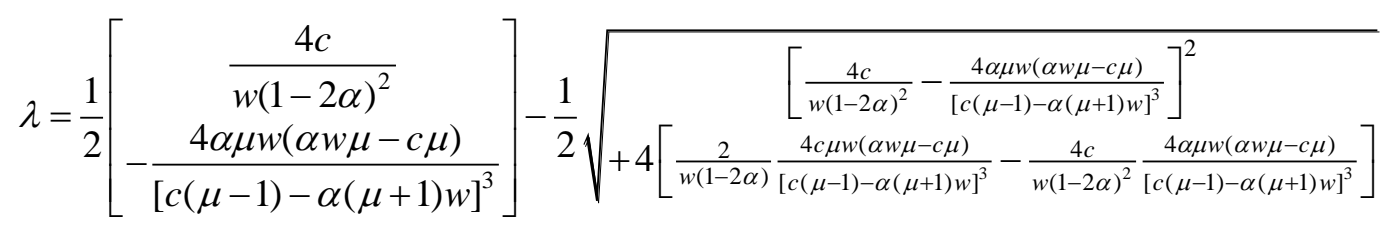

Given $(1,0), \lambda<0$ resumes to verifying

$$
\frac{4 c}{w(1-2 \alpha)^{2}} \geq \frac{4 \alpha \mu w(\alpha w \mu-c \mu)}{[c(\mu-1)-\alpha(\mu+1) w]^{3}}
$$

and

$$
\frac{2}{w(1-2 \alpha)} \frac{4 c \mu w(\alpha w \mu-c \mu)}{[c(\mu-1)-\alpha(\mu+1) w]^{3}}>\frac{4 c}{w(1-2 \alpha)^{2}} \frac{4 \alpha \mu w(\alpha w \mu-c \mu)}{[c(\mu-1)-\alpha(\mu+1) w]^{3}}
$$


Table 6 Eigenvalues of the Jacobian matrix with $c=\frac{1}{2} w$ for $S=(1,0)$

\begin{tabular}{l|ccccccccccccc|}
$\alpha / \mu$ & 0.00 & 0.01 & 0.10 & 0.20 & 0.30 & 0.40 & 0.50 & 0.60 & 0.70 & 0.80 & 0.90 & 0.99 & 1.00 \\
\cline { 2 - 11 } 0.00 & 0.00 & -0.00 & -0.00 & -0.00 & -0.00 & -0.00 & -0.00 & -0.00 & -0.00 & -0.01 & -0.01 & -0.01 & \\
0.01 & 0.00 & -0.00 & -0.00 & -0.00 & -0.00 & -0.00 & -0.00 & -0.00 & -0.00 & -0.00 & -0.01 & -0.02 & $-\mathbf{3 6 . 8}$ \\
0.10 & 0.00 & -0.00 & -0.00 & -0.00 & -0.00 & -0.00 & -0.00 & -0.00 & -0.00 & -0.00 & -0.00 & -0.00 & -0.00 \\
0.20 & 0.00 & -0.00 & -0.00 & -0.00 & -0.00 & -0.00 & -0.00 & -0.00 & -0.00 & -0.00 & -0.00 & -0.00 & -0.00 \\
0.30 & 0.00 & -0.00 & -0.00 & -0.00 & -0.00 & -0.00 & -0.00 & -0.00 & -0.00 & -0.00 & -0.00 & -0.00 & -0.00 \\
0.40 & 0.00 & -0.00 & -0.00 & -0.00 & -0.00 & -0.00 & -0.00 & -0.00 & -0.00 & -0.00 & -0.00 & -0.00 & -0.00 \\
0.50 & & & & & & & & & & & & & \\
0.60 & 0.00 & -0.00 & -0.00 & -0.00 & -0.00 & -0.00 & -0.00 & -0.00 & -0.00 & -0.00 & -0.00 & -0.00 & -0.00 \\
0.70 & 0.00 & -0.00 & -0.00 & -0.00 & -0.00 & -0.00 & -0.00 & -0.00 & -0.00 & -0.00 & -0.00 & -0.00 & -0.00 \\
0.80 & 0.00 & -0.00 & -0.00 & -0.00 & -0.00 & -0.00 & -0.00 & -0.00 & -0.00 & -0.00 & -0.00 & -0.00 & -0.00 \\
0.90 & 0.00 & -0.00 & -0.00 & -0.00 & -0.00 & -0.00 & -0.00 & -0.00 & -0.00 & -0.00 & -0.00 & -0.00 & -0.00 \\
0.99 & 0.00 & -0.00 & -0.00 & -0.00 & -0.00 & -0.00 & -0.00 & -0.00 & -0.00 & -0.00 & -0.00 & -0.00 & -0.00 \\
1.00 & 0.00 & -0.00 & -0.00 & -0.00 & -0.00 & -0.00 & -0.00 & -0.00 & -0.00 & -0.00 & -0.00 & -0.00 & -0.00 \\
\hline
\end{tabular}


- For $S=(1,1)$

$$
J(\alpha, c)=\left[\begin{array}{cc}
\frac{4 c}{w(1-2 \alpha)^{2}}-\lambda & \frac{2}{w(1-2 \alpha)} \\
\frac{4 c \mu w(\alpha w+c)}{[c(\mu-1)-\alpha(\mu+1) w]^{3}} & -\frac{4 \alpha \mu w(\alpha w+c)}{[c(\mu-1)-\alpha(\mu+1) w]^{3}}-\lambda
\end{array}\right]
$$

So

$\operatorname{det}[J(\alpha, c)]=\left[\frac{4 c}{w(1-2 \alpha)^{2}}-\lambda\right]\left[-\frac{4 \alpha \mu w(\alpha w+c)}{[c(\mu-1)-\alpha(\mu+1) w]^{3}}-\lambda\right]-\left[\frac{2}{w(1-2 \alpha)}\right]\left[\frac{4 c \mu w(\alpha w+c)}{[c(\mu-1)-\alpha(\mu+1) w]^{3}}\right]$

And

$$
\left[\frac{4 c}{w(1-2 \alpha)^{2}}-\lambda\right]\left[-\frac{4 \alpha \mu w(\alpha w+c)}{[c(\mu-1)-\alpha(\mu+1) w]^{3}}-\lambda\right]-\left[\frac{2}{w(1-2 \alpha)}\right]\left[\frac{4 c \mu w(\alpha w+c)}{[c(\mu-1)-\alpha(\mu+1) w]^{3}}\right]=0
$$

Which gives

$$
\lambda=\frac{1}{2}\left[\begin{array}{c}
\frac{4 c}{w(1-2 \alpha)^{2}} \\
-\frac{4 \alpha \mu w(\alpha w+c)}{[c(\mu-1)-\alpha(\mu+1) w]^{3}}
\end{array}\right]-\frac{1}{2} \sqrt{+4\left[\frac{2}{w(1-2 \alpha)} \frac{\left[\frac{4 c}{w(1-2 \alpha)^{2}}-\frac{4 \alpha \mu w(\alpha w+c)}{[c(\mu-1)-\alpha(\mu+1) w]^{3}}-\frac{4 c}{w(1-2 \alpha)^{2}} \frac{4 \alpha \mu w(\alpha w+c)}{[c(\mu-1)-\alpha(\mu+1) w]^{3}}\right.}{[(\mu)+1) w]^{3}}\right]^{2}}
$$

Given $(1,1), \lambda<0$ resumes to verifying

$$
\frac{4 c}{w(1-2 \alpha)^{2}} \geq \frac{4 \alpha \mu w(\alpha w+c)}{[c(\mu-1)-\alpha(\mu+1) w]^{3}}
$$

And

$$
\frac{2}{w(1-2 \alpha)} \frac{4 c \mu w(\alpha w+c)}{[c(\mu-1)-\alpha(\mu+1) w]^{3}}>\frac{4 c}{w(1-2 \alpha)^{2}} \frac{4 \alpha \mu w(\alpha w+c)}{[c(\mu-1)-\alpha(\mu+1) w]^{3}}
$$


Table 7 Eigenvalues of the Jacobian matrix with $c=\frac{1}{2} w$ for $S=(1,1)$

\begin{tabular}{llllllllllllll|}
$\alpha / \mu$ & 0.00 & 0.01 & 0.10 & 0.20 & 0.30 & 0.40 & 0.50 & 0.60 & 0.70 & 0.80 & 0.90 & 0.99 & 1.00 \\
\cline { 2 - 12 } 0.00 & 0.00 & +0.00 & +0.00 & +0.00 & +0.00 & +0.00 & +0.00 & +0.00 & +0.01 & +0.01 & +0.01 & +0.01 & \\
0.01 & 0.00 & +0.00 & +0.00 & +0.00 & +0.00 & +0.00 & +0.00 & -0.01 & -0.03 & -0.15 & $-\mathbf{1 . 6 7}$ & $\mathbf{- 2 4 0}$ & $\mathbf{- 8 9 3}$ \\
0.10 & 0.00 & -0.00 & -0.01 & -0.01 & -0.03 & -0.06 & -0.13 & -0.25 & -0.53 & $\mathbf{- 1 . 2 1}$ & $-\mathbf{3 . 1 5}$ & $\mathbf{- 9 . 1 6}$ & $\mathbf{- 1 0 . 5}$ \\
0.20 & 0.00 & -0.00 & -0.02 & -0.04 & -0.08 & -0.15 & -0.24 & -0.39 & -0.64 & $-\mathbf{1 . 0 4}$ & $-\mathbf{- 1 . 7 5}$ & $-\mathbf{2 . 8 9}$ & $\mathbf{- 3 . 0 6}$ \\
0.30 & 0.00 & -0.00 & -0.03 & -0.08 & -0.13 & -0.21 & -0.30 & -0.43 & -0.60 & -0.83 & $-\mathbf{1 . 1 4}$ & $-\mathbf{1 . 5 1}$ & $-\mathbf{1 . 5 6}$ \\
0.40 & 0.00 & -0.00 & -0.05 & -0.11 & -0.18 & -0.25 & -0.34 & -0.43 & -0.55 & -0.67 & -0.82 & -0.97 & -0.98 \\
0.50 & & & & & & & & & & & & & \\
0.60 & 0.00 & -0.01 & -0.09 & -0.17 & -0.24 & -0.30 & -0.35 & -0.40 & -0.44 & -0.48 & -0.51 & -0.53 & -0.53 \\
0.70 & 0.00 & -0.01 & -0.11 & -0.20 & -0.26 & -0.31 & -0.35 & -0.38 & -0.40 & -0.41 & -0.42 & -0.43 & -0.43 \\
0.80 & 0.00 & -0.02 & -0.13 & -0.22 & -0.28 & -0.32 & -0.34 & -0.36 & -0.36 & -0.37 & -0.36 & -0.36 & -0.36 \\
0.90 & 0.00 & -0.02 & -0.15 & -0.24 & -0.30 & -0.32 & -0.34 & -0.34 & -0.33 & -0.33 & -0.31 & -0.30 & -0.30 \\
0.99 & 0.00 & -0.02 & -0.17 & -0.26 & -0.31 & -0.32 & -0.33 & -0.32 & -0.31 & -0.30 & -0.28 & -0.27 & -0.27 \\
1.00 & 0.00 & -0.02 & -0.17 & -0.26 & -0.31 & -0.32 & -0.33 & -0.32 & -0.31 & -0.29 & -0.28 & -0.26 & -0.26 \\
\hline
\end{tabular}


- For $S=(s, r)$

$$
J(\alpha, c)=\left[\begin{array}{cc}
\frac{4 c s}{w(1-2 \alpha)^{2}}-\lambda & \frac{2 s}{w(1-2 \alpha)} \\
\frac{4 c \mu w[\alpha w(\mu-\mu r+r)+c(-\mu+\mu r+r)]}{[c(\mu-1)-\alpha(\mu+1) w]^{3}} & -\frac{4 \alpha \mu w[\alpha w(\mu-\mu r+r)+c(-\mu+\mu r+r)]}{[c(\mu-1)-\alpha(\mu+1) w]^{3}}-\lambda
\end{array}\right]
$$

So

$$
\begin{aligned}
\operatorname{det}[J(\alpha, c)] & =\left[\frac{4 c s}{w(1-2 \alpha)^{2}}-\lambda\right]\left[-\frac{4 \alpha \mu w[\alpha w(\mu-\mu r+r)+c(-\mu+\mu r+r)]}{[c(\mu-1)-\alpha(\mu+1) w]^{3}}-\lambda\right] \\
& -\left[\frac{2 s}{w(1-2 \alpha)}\right]\left[\frac{4 c \mu w[\alpha w(\mu-\mu r+r)+c(-\mu+\mu r+r)]}{[c(\mu-1)-\alpha(\mu+1) w]^{3}}\right]
\end{aligned}
$$

And

$$
\begin{gathered}
{\left[\frac{4 c s}{w(1-2 \alpha)^{2}}-\lambda\right]\left[-\frac{4 \alpha \mu w[\alpha w(\mu-\mu r+r)+c(-\mu+\mu r+r)]}{[c(\mu-1)-\alpha(\mu+1) w]^{3}}-\lambda\right]} \\
-\left[\frac{2 s}{w(1-2 \alpha)}\right]\left[\frac{4 c \mu w[\alpha w(\mu-\mu r+r)+c(-\mu+\mu r+r)]}{[c(\mu-1)-\alpha(\mu+1) w]^{3}}\right]=0
\end{gathered}
$$

which gives

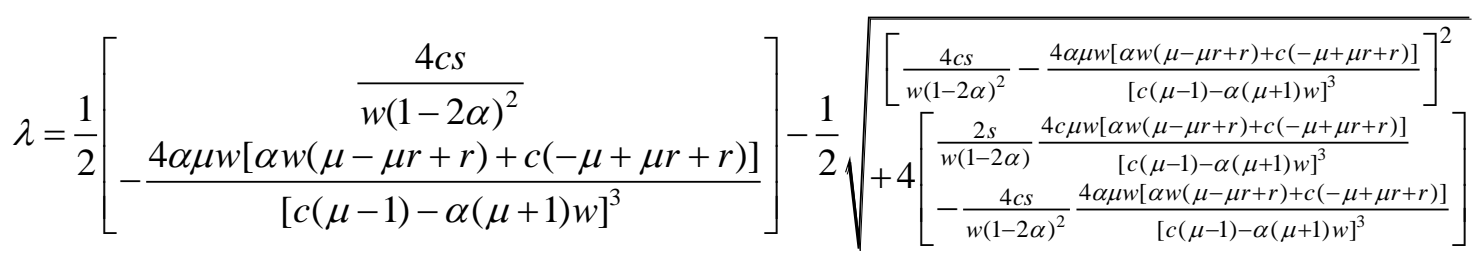

We know that $s^{*}=\frac{\tilde{q}-2}{2(\tilde{q}-1)}, r^{*}=\frac{\tilde{p}-2}{2(\tilde{p}-1)}$. Given $\left(s^{*}, r^{*}\right), \lambda<0$ resumes to verifying

$$
\frac{4 c s^{*}}{w(1-2 \alpha)^{2}} \geq \frac{4 \alpha \mu w\left[\alpha w\left(\mu-\mu r^{*}+r^{*}\right)+c\left(-\mu+\mu r^{*}+r^{*}\right)\right]}{[c(\mu-1)-\alpha(\mu+1) w]^{3}}
$$

And

$$
\begin{gathered}
\frac{2 s^{*}}{w(1-2 \alpha)} \frac{4 c \mu w\left[\alpha w\left(\mu-\mu r^{*}+r^{*}\right)+c\left(-\mu+\mu r^{*}+r^{*}\right)\right]}{[c(\mu-1)-\alpha(\mu+1) w]^{3}} \\
-\frac{4 c s^{*}}{w(1-2 \alpha)^{2}} \frac{4 \alpha \mu w\left[\alpha w\left(\mu-\mu r^{*}+r^{*}\right)+c\left(-\mu+\mu r^{*}+r^{*}\right)\right]}{[c(\mu-1)-\alpha(\mu+1) w]^{3}}>0
\end{gathered}
$$


Table 8 Eigenvalues of the Jacobian matrix with $c=\frac{1}{2} w$ for $S=\left(s^{*}, r^{*}\right)$

\begin{tabular}{l|lllllllllllll|}
$\alpha / \mu$ & 0.00 & 0.01 & 0.10 & 0.20 & 0.30 & 0.40 & 0.50 & 0.60 & 0.70 & 0.80 & 0.90 & 0.99 & 1.00 \\
0.00 & 0.00 & \pm & \pm & \pm & \pm & \pm & \pm & \pm & \pm & \pm & \pm & \pm & \pm \\
0.01 & 0.00 & \pm & \pm & \pm & \pm & \pm & \pm & \pm & \pm & \pm & \pm & \pm & \pm \\
0.10 & 0.00 & \pm & \pm & \pm & \pm & \pm & \pm & \pm & \pm & \pm & \pm & \pm & \pm \\
0.20 & 0.00 & \pm & \pm & \pm & \pm & \pm & \pm & \pm & \pm & \pm & \pm & \pm & \pm \\
0.30 & 0.00 & \pm & \pm & \pm & \pm & \pm & \pm & \pm & \pm & \pm & \pm & \pm & \pm \\
0.40 & 0.00 & \pm & \pm & \pm & \pm & \pm & \pm & \pm & \pm & \pm & \pm & \pm & \pm \\
0.50 & & & & & & & & & & & & & \\
0.60 & 0.00 & - & - & - & - & - & - & - & - & - & - & - & - \\
0.70 & 0.00 & - & - & - & - & - & - & - & - & - & - & - & - \\
0.80 & 0.00 & - & - & - & - & - & - & - & - & - & - & - & - \\
0.90 & 0.00 & - & - & - & - & - & - & - & - & - & - & - & - \\
0.99 & 0.00 & - & - & - & - & - & - & - & - & - & - & - & - \\
1.00 & 0.00 & - & - & - & - & - & - & - & - & - & - & - & - \\
\cline { 2 - 9 }
\end{tabular}

